Supporting Information

\title{
Fast Modulation of Surface Amphiphobicity/Amphiphilicity via Bidirectional Substitution between Perfluorinated Surfactant and Polyanions throughout Pre-Assembled Polyelectrolyte Multilayers
}

Hao Zhang, ${ }^{\dagger}$ Li Yu, ${ }^{*},{ }^{\dagger}$ Linbo Han, ${ }^{\dagger}$ Xiaokong Liu, ${ }^{\star}$ Shuangchen Ruan, ${ }^{*}{ }^{\dagger}$ and Junqing $\mathrm{Hu}^{\dagger}$

$\dagger$ College of Health Science and Environmental Engineering, Shenzhen Technology University, Shenzhen, 518118, China

$\$$ State Key Laboratory of Supramolecular Structure and Materials, College of Chemistry, Jilin University, Changchun, 130012, China

*Address correspondence to yuli@sztu.edu.cn; ruanshuangchen@sztu.edu.cn 




Figure S1. UV-Vis absorption spectra of (PDDA/PSS) ${ }_{n}$ PEMs with $n$ ranging from 1 to 7. The PEMs were prepared via LbL assembly in the presence of $1.0 \mathrm{M} \mathrm{NaCl}$. 

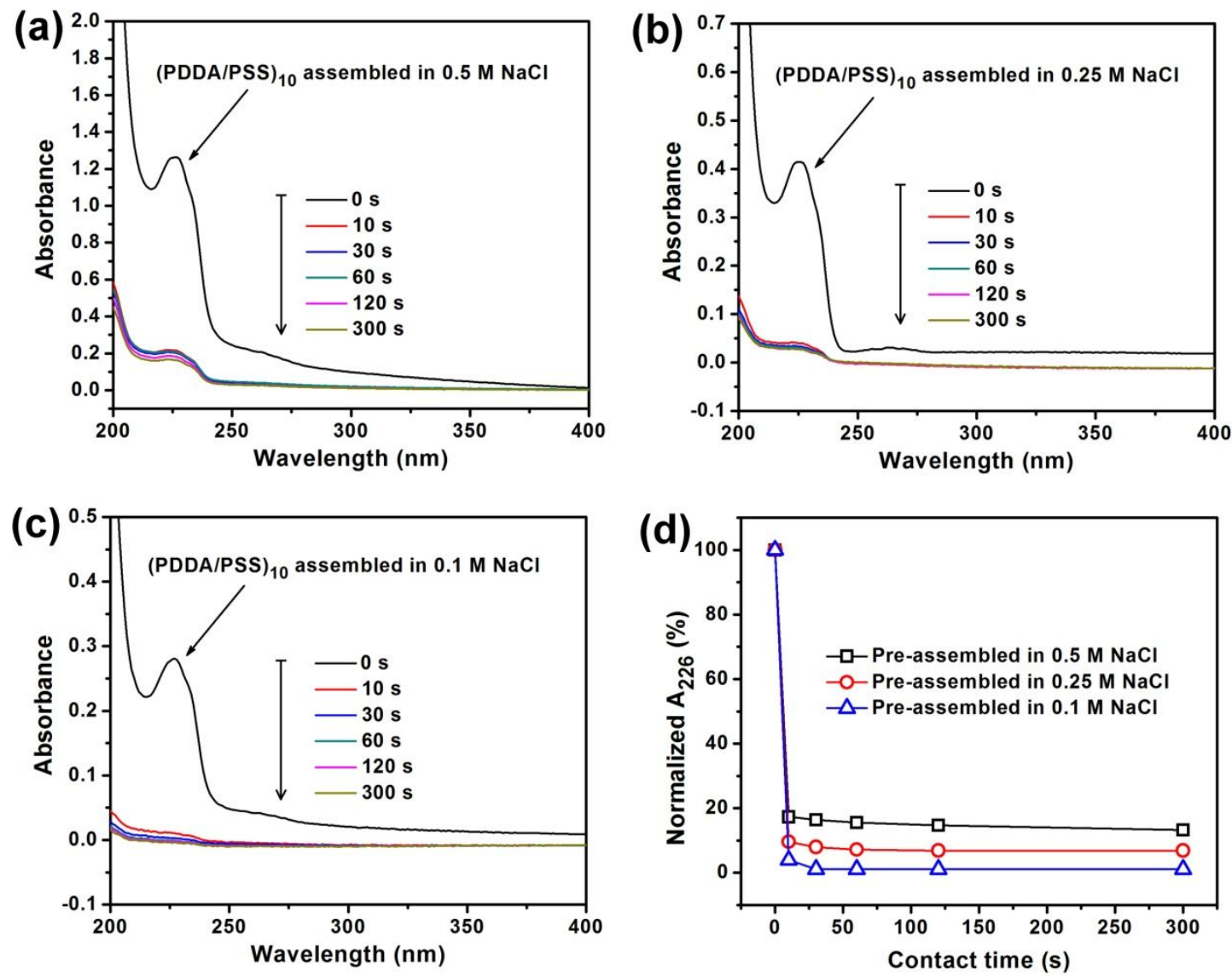

Figure S2. (a-c) UV-Vis absorption spectra of the (PDDA/PSS) $)_{10}$ PEMs prepared in the presence of $0.5 \mathrm{M}(\mathrm{a}), 0.25 \mathrm{M}$ (b) and $0.1 \mathrm{M}$ (c) $\mathrm{NaCl}$, before (black curves) and after (colored curves) the incubation in $50 \mathrm{mM}$ PFO aqueous solutions for different contact times specified in the figure legends. (d) Normalized $\mathrm{A}_{226}$ of (PDDA/PSS) ${ }_{10}$ PEMs prepared in the presence of $0.5 \mathrm{M}$ (black squares), $0.25 \mathrm{M}$ (red circles) and $0.1 \mathrm{M}$ (blue triangles) $\mathrm{NaCl}$ versus the contact time used for the incubation of the PEMs in $50 \mathrm{mM}$ PFO solutions. 
Table S1. Summary of the atomic concentrations of the various elements on the $(\mathrm{PDDA} / \mathrm{PSS})_{7}$ PEMs $(0 \mathrm{~s})$ and those incubated in $50 \mathrm{mM}$ PFO solution with incubation time of $2 \mathrm{~s}, 5 \mathrm{~s}, 10 \mathrm{~s}, 30 \mathrm{~s}, 60 \mathrm{~s}, 120 \mathrm{~s}$ and $300 \mathrm{~s}$, respectively. The atomic concentrations were deduced from the XPS spectra of the corresponding samples. The (PDDA/PSS $)_{7}$ PEMs were prepared via LbL assembly in the presence of $1.0 \mathrm{M} \mathrm{NaCl}$.

\begin{tabular}{ccccccc}
\hline \multirow{2}{*}{$\begin{array}{c}\text { Contact time } \\
(\mathrm{s})\end{array}$} & $\mathrm{C}$ & $\mathrm{N}$ & $\mathrm{O}$ & $\mathrm{F}$ & $\mathrm{S}$ & $\mathrm{Na}$ \\
\cline { 2 - 7 } & 71.72 & 3.08 & 17.35 & 0 & 4.59 & 3.26 \\
0 & 51.72 & 3.42 & 8.35 & 35.22 & 1.29 & 0 \\
2 & 49.43 & 3.29 & 7.32 & 38.99 & 0.97 & 0 \\
5 & 48.74 & 3.32 & 7.00 & 40.13 & 0.81 & 0 \\
10 & 48.40 & 3.33 & 6.80 & 40.70 & 0.77 & 0 \\
30 & 48.29 & 3.38 & 6.61 & 41.01 & 0.71 & 0 \\
60 & 47.43 & 3.34 & 6.49 & 42.06 & 0.68 & 0 \\
120 & 47.21 & 3.36 & 6.46 & 42.34 & 0.63 & 0 \\
300 & & & & & & \\
\hline
\end{tabular}

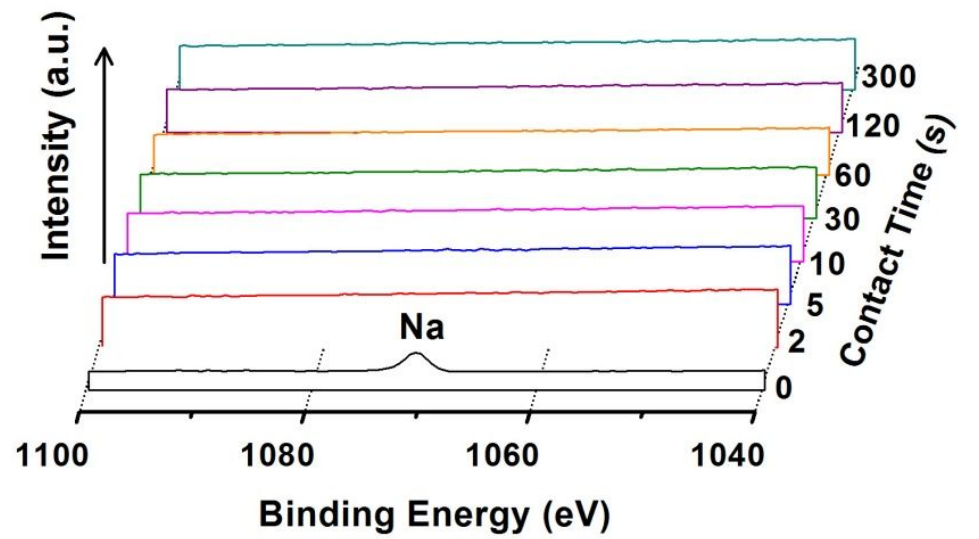

Figure S3. High-resolution XPS spectra of $\mathrm{Na}$ element collected from the original $(\mathrm{PDDA} / \mathrm{PSS})_{7}$ PEMs $(0 \mathrm{~s})$ and those incubated in $50 \mathrm{mM}$ PFO solution with incubation time of $2 \mathrm{~s}, 5 \mathrm{~s}, 10 \mathrm{~s}, 30 \mathrm{~s}, 60 \mathrm{~s}, 120 \mathrm{~s}$ and $300 \mathrm{~s}$, respectively. 

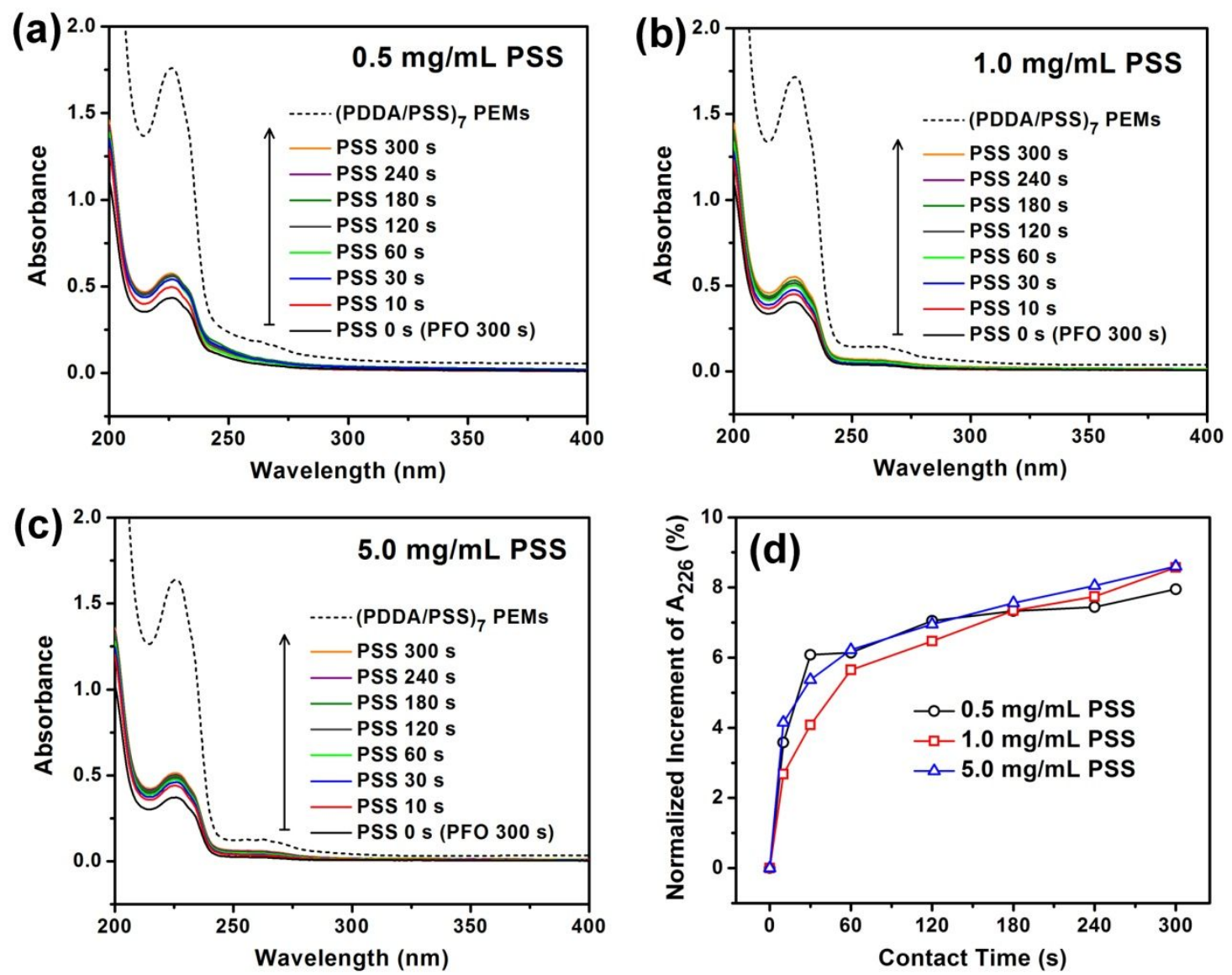

Figure S4. (a-c) UV-Vis absorption spectra of the PFO-(PDDA/PSS) ${ }_{7}$ films before (black curve) and after (colored curves) incubation in PSS solutions with different contact times specified in the figure legends. The concentrations of the PSS solutions are respectively 0.5 $\mathrm{mg} / \mathrm{mL}$ (a), $1.0 \mathrm{mg} / \mathrm{mL}$ (b) and $5.0 \mathrm{mg} / \mathrm{mL}$ (c). (d) Normalized increment of $\mathrm{A}_{226}$ of the PFO$(\mathrm{PDDA} / \mathrm{PSS})_{7}$ films versus the contact time used for the incubation of the films in PSS solutions at concentrations of $0.5 \mathrm{mg} / \mathrm{mL}$ (black circles), $1.0 \mathrm{mg} / \mathrm{mL}$ (red squares) and 5.0 $\mathrm{mg} / \mathrm{mL}$ (blue triangles). The original (PDDA/PSS) ${ }_{7}$ PEMs were prepared via LbL assembly in the presence of $1.0 \mathrm{M} \mathrm{NaCl}$ (black dash lines), the PFO-(PDDA/PSS) ${ }_{7}$ films were obtained by incubation of the (PDDA/PSS) 7 PEMs in $50 \mathrm{mM}$ PFO solutions for $300 \mathrm{~s}$ (black lines). 

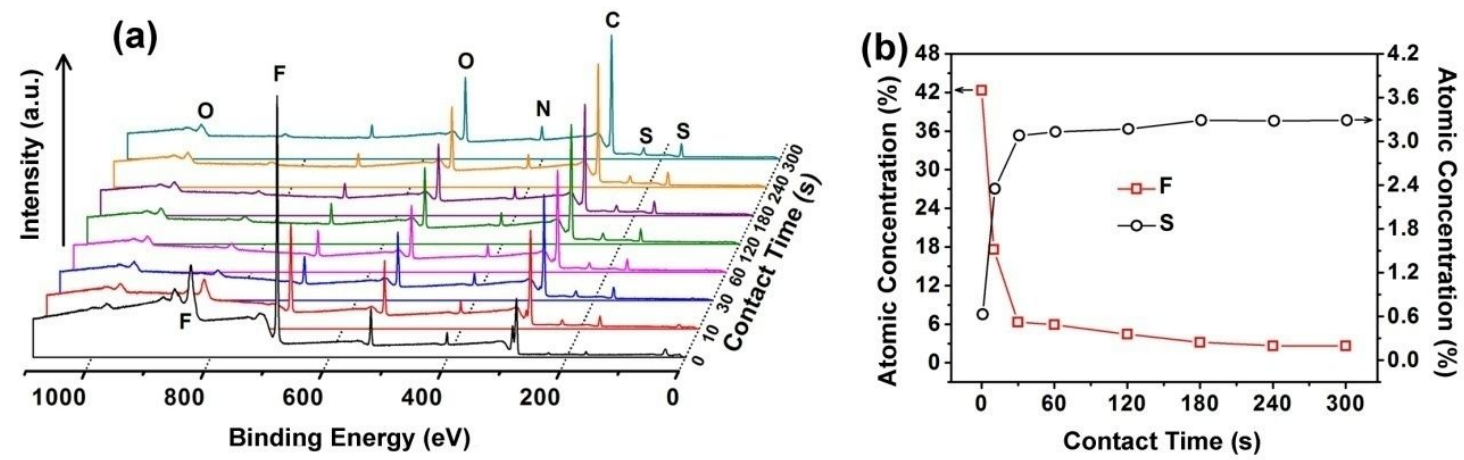

Figure S5. (a) XPS wide scan spectra of the PFO-(PDDA/PSS $)_{7}$ films $(0 \mathrm{~s})$ and those films incubated in $1 \mathrm{mg} / \mathrm{mL}$ PSS solutions with contact time varies from 10, 30, 60, 120, 180, 240 to $300 \mathrm{~s}$. (b) Plots of the atomic concentrations of $\mathrm{F}$ and $\mathrm{S}$ on the films versus the contact time of PSS treatment. The atomic concentrations were deduced from the XPS spectra of the corresponding samples.

Table S2. Summary of the atomic concentrations of the various elements on the PFO$(\mathrm{PDDA} / \mathrm{PSS})_{7}$ films $(0 \mathrm{~s})$ and those incubated in $1 \mathrm{mg} / \mathrm{mL}$ PSS solution with incubation time of $10 \mathrm{~s}, 30 \mathrm{~s}, 60 \mathrm{~s}, 120 \mathrm{~s}, 180 \mathrm{~s}, 240 \mathrm{~s}$ and $300 \mathrm{~s}$, respectively. The atomic concentrations were deduced from the XPS spectra of the corresponding samples. The (PDDA/PSS) 7 PEMs were prepared via LbL assembly in the presence of $1.0 \mathrm{M} \mathrm{NaCl}$. The PFO-(PDDA/PSS) 7 films were obtained by incubation of (PDDA/PSS) ${ }_{7}$ PEMs in $50 \mathrm{mM}$ PFO solutions for $300 \mathrm{~s}$.

\begin{tabular}{ccccccc}
\hline \multirow{2}{*}{$\begin{array}{c}\text { Contact time } \\
(\mathrm{s})\end{array}$} & $\mathrm{C}$ & $\mathrm{N}$ & $\mathrm{O}$ & $\mathrm{F}$ & $\mathrm{S}$ & $\mathrm{Na}$ \\
\cline { 2 - 7 } & 47.21 & 3.36 & 6.46 & 42.34 & 0.63 & 0 \\
0 & 64.13 & 4.07 & 11.84 & 17.61 & 2.35 & 0 \\
10 & 72.28 & 4.50 & 13.80 & 6.34 & 3.08 & 0 \\
30 & 72.80 & 4.30 & 13.85 & 5.92 & 3.13 & 0 \\
60 & 74.16 & 4.27 & 13.95 & 4.45 & 3.17 & 0 \\
120 & 74.98 & 4.43 & 14.12 & 3.18 & 3.29 & 0 \\
180 & 75.09 & 4.67 & 14.31 & 2.65 & 3.28 & 0 \\
240 & 75.26 & 4.49 & 14.33 & 2.63 & 3.29 & 0 \\
300 & \multicolumn{7}{c}{}
\end{tabular}




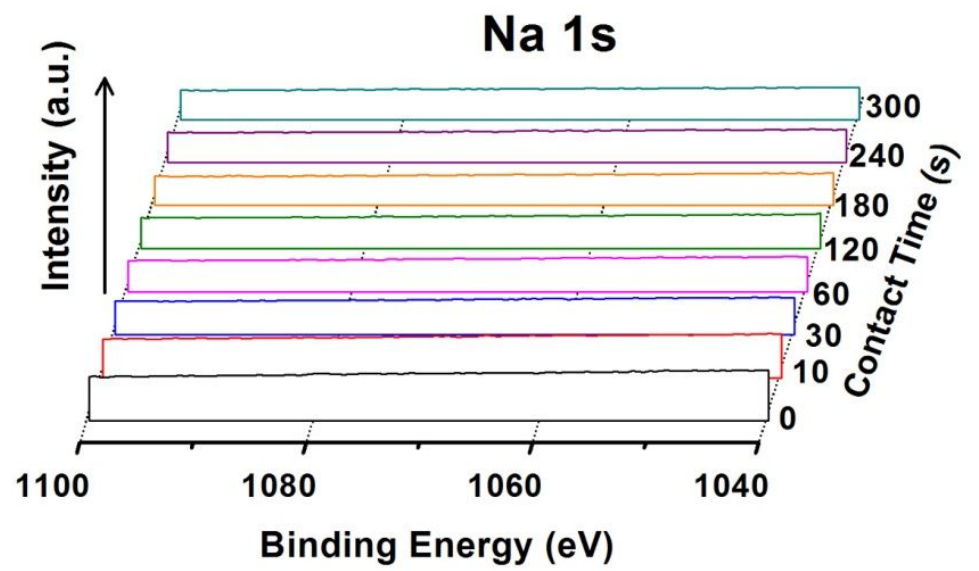

Figure S6. High-resolution XPS spectra of $\mathrm{Na}$ element collected from the PFO$(\mathrm{PDDA} / \mathrm{PSS})_{7}$ films $(0 \mathrm{~s})$ and those incubated in $1 \mathrm{mg} / \mathrm{mL}$ PSS solution with incubation time of $10 \mathrm{~s}, 30 \mathrm{~s}, 60 \mathrm{~s}, 120 \mathrm{~s}, 60 \mathrm{~s}, 120 \mathrm{~s}, 180 \mathrm{~s}, 240 \mathrm{~s}$ and $300 \mathrm{~s}$, respectively. It can be observed that no signals of $\mathrm{Na}$ element were detected on the films surface.

Table S3. Summary of the atomic concentrations of the various elements on the PFO$(\mathrm{PDDA} / \mathrm{PSS})_{7}$ films $(0 \mathrm{~s})$ and those incubated in $1 \mathrm{M} \mathrm{NaCl}$-containing PSS $(1 \mathrm{mg} / \mathrm{mL})$ solution with incubation time of $5 \mathrm{~s}, 10 \mathrm{~s}, 30 \mathrm{~s}, 60 \mathrm{~s}, 120 \mathrm{~s}, 300 \mathrm{~s}, 600 \mathrm{~s}$ and $900 \mathrm{~s}$, respectively. The atomic concentrations were deduced from the XPS spectra of the corresponding samples. The (PDDA/PSS) ${ }_{7}$ PEMs were prepared via LbL assembly in the presence of $1.0 \mathrm{M} \mathrm{NaCl}$. The PFO-(PDDA/PSS) 7 films were obtained by incubation of $(\mathrm{PDDA} / \mathrm{PSS})_{7}$ PEMs in $50 \mathrm{mM}$ PFO solutions for $300 \mathrm{~s}$.

\begin{tabular}{ccccccc}
\hline \multirow{2}{*}{$\begin{array}{c}\text { Contact time } \\
(\mathrm{s})\end{array}$} & $\mathrm{C}$ & $\mathrm{N}$ & $\mathrm{O}$ & $\mathrm{F}$ & $\mathrm{S}$ & $\mathrm{Na}$ \\
\cline { 2 - 7 } & 47.21 & 3.36 & 6.46 & 42.34 & 0.63 & 0 \\
0 & 66.54 & 4.36 & 13.04 & 13.19 & 2.87 & 0 \\
5 & 68.47 & 4.57 & 14.31 & 9.43 & 3.22 & 0 \\
10 & 70.18 & 4.86 & 18.31 & 2.03 & 3.84 & 0.78 \\
30 & 72.73 & 4.36 & 16.35 & 1.32 & 4.29 & 0.95 \\
60 & 69.01 & 4.32 & 19.17 & 1.34 & 4.58 & 1.58 \\
120 & 69.04 & 4.09 & 18.67 & 1.82 & 4.65 & 1.73 \\
300 & 68.23 & 3.58 & 19.23 & 1.88 & 4.75 & 2.33 \\
600 & 68.27 & 3.60 & 19.68 & 1.36 & 4.82 & 2.27 \\
900 & & & & & &
\end{tabular}




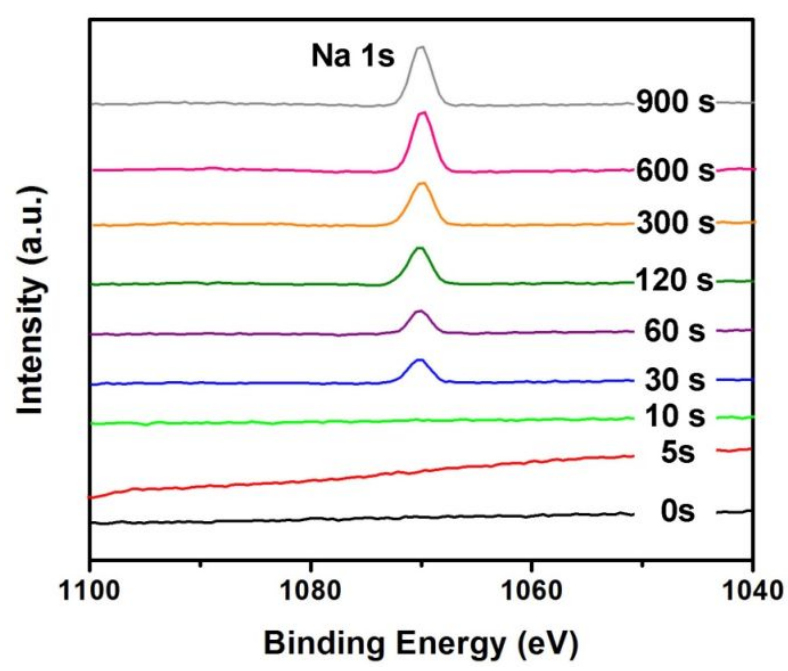

Figure S7. High-resolution XPS spectra of $\mathrm{Na}$ element collected from the PFO$(\mathrm{PDDA} / \mathrm{PSS})_{7}$ films $(0 \mathrm{~s})$ and those incubated in $1 \mathrm{M} \mathrm{NaCl}$-containing PSS $(1 \mathrm{mg} / \mathrm{mL})$ solution with incubation time of $5 \mathrm{~s}, 10 \mathrm{~s}, 30 \mathrm{~s}, 60 \mathrm{~s}, 120 \mathrm{~s}, 300 \mathrm{~s}, 600 \mathrm{~s}$ and $900 \mathrm{~s}$, respectively.

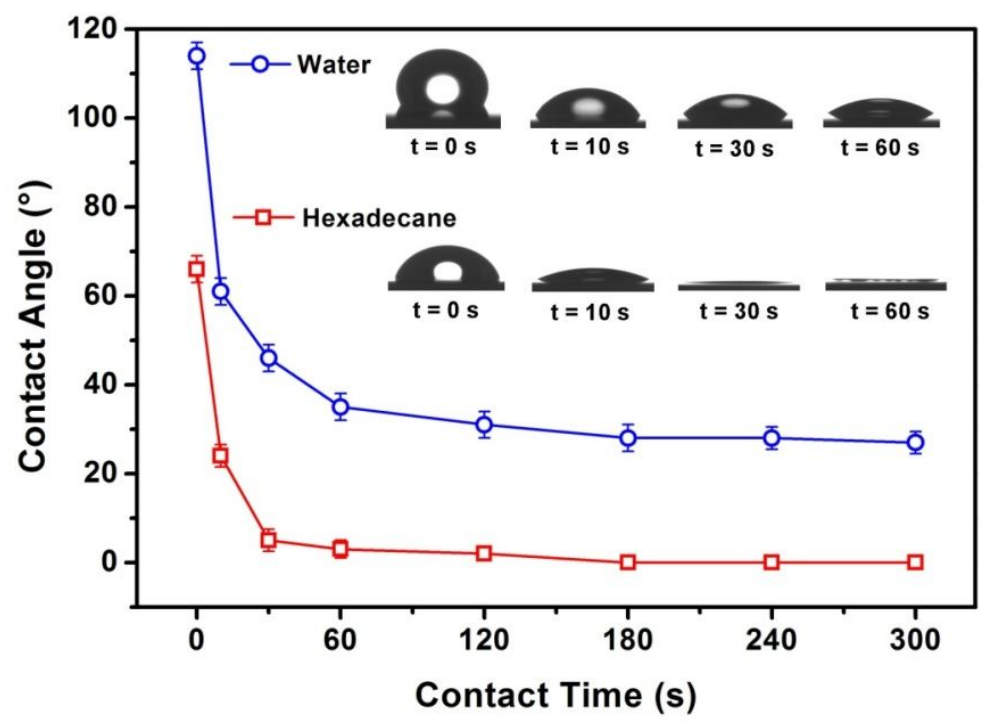

Figure S8. CAs of water (blue circles) and hexadecane (red squares) droplets on the $\mathrm{Si}$ substrate-supported PFO-(PDDA/PSS $)_{7}$ film coatings as a function of the contact time used for the incubation of the film coatings in $1.0 \mathrm{mg} / \mathrm{mL}$ PSS solutions. Insets show the shape profiles of the $2 \mu \mathrm{L}$ water and hexadecane droplets on the Si substrate-supported PFO$(\mathrm{PDDA} / \mathrm{PSS})_{7}$ film coatings with different contact times. 

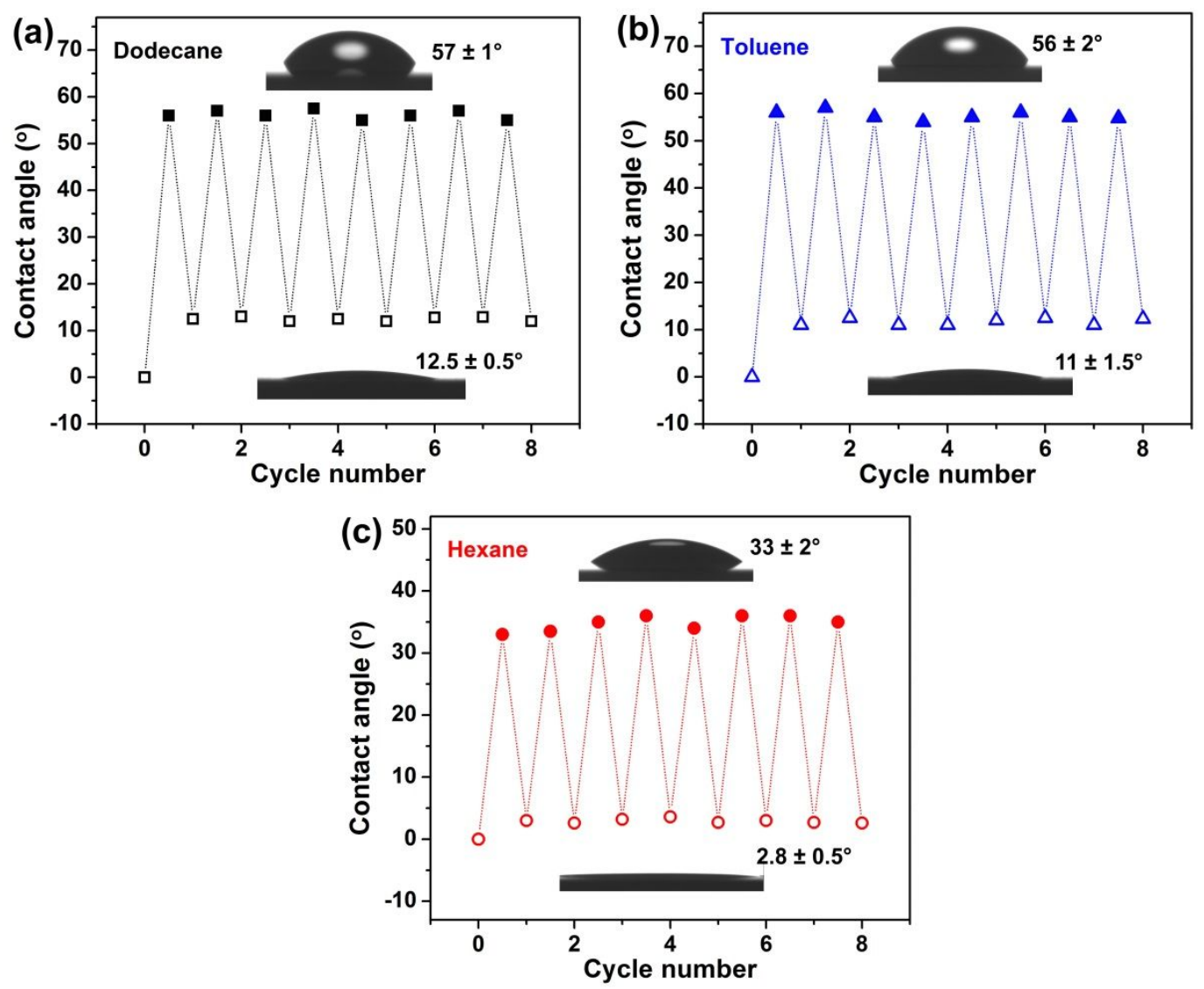

Figure S9. CAs of dodecane (a), toluene (b) and hexane (c) on the Si substrate-supported $(\mathrm{PDDA} / \mathrm{PSS})_{7}$ films coatings (cycle number 0 ) over 8 cycles incubation in $50 \mathrm{mM}$ PFO (closed symbols) and 1.0 M NaCl-containing PSS (open symbols) solutions, respectively. The original (PDDA/PSS) ${ }_{7}$ PEMs were prepared via LbL assembly in the presence of $1.0 \mathrm{M}$ $\mathrm{NaCl}$. The incubation times of the film in the PFO and PSS solutions are $2 \mathrm{~s}$ and $10 \mathrm{~s}$, respectively. Insets show the shape profiles of the $2 \mu \mathrm{L}$ probe liquids on the corresponding samples. 

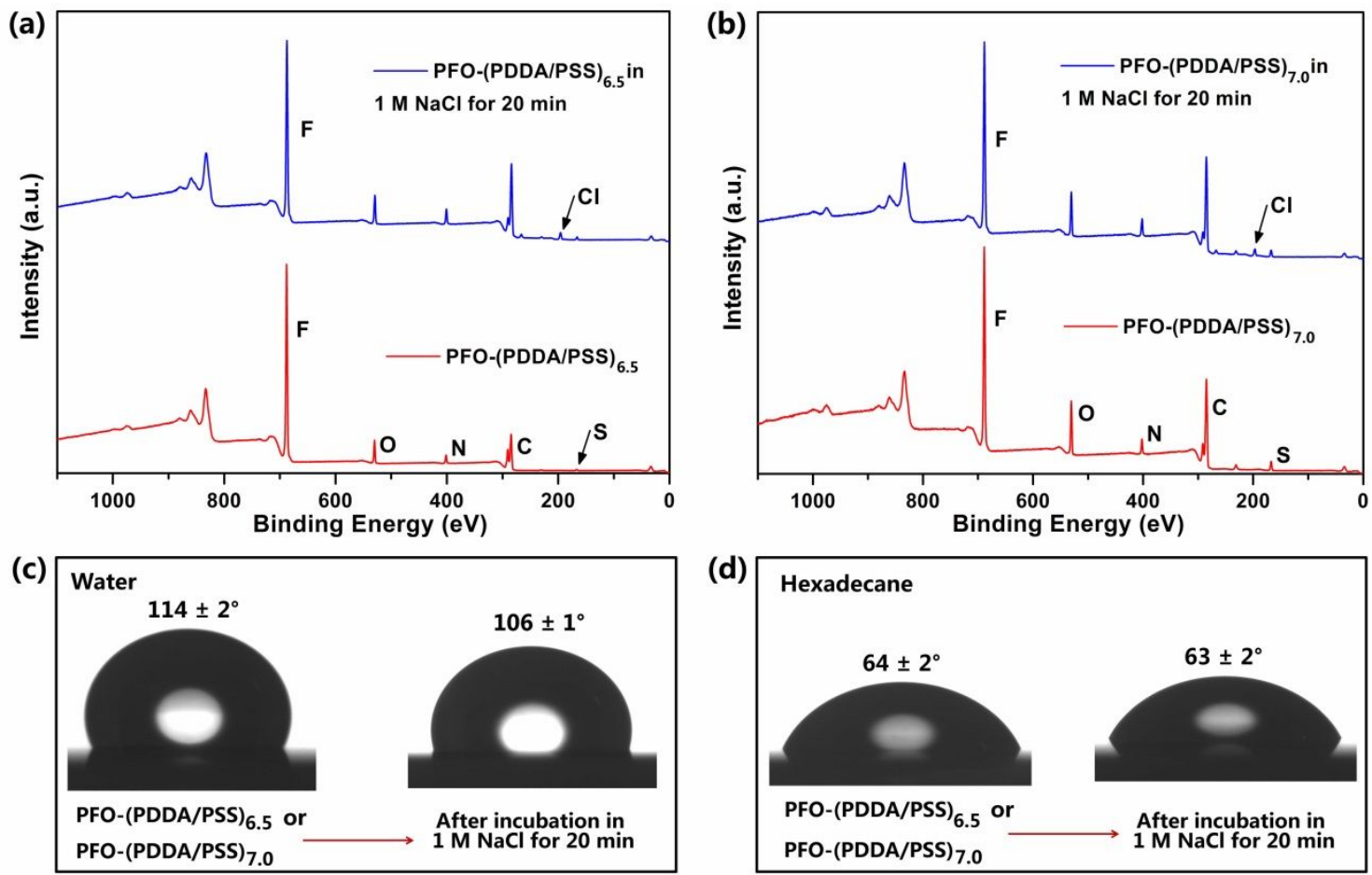

Figure S10. (a, b) XPS wide scan spectra of the PFO-(PDDA/PSS) 6.5 (a) and PFO$(\mathrm{PDDA} / \mathrm{PSS})_{7}$ (b) films (red lines) and those after incubation in $1 \mathrm{M} \mathrm{NaCl}$ solution for $20 \mathrm{~min}$ (blue lines). (c, d) CAs of water (c) and hexadecane (d) droplets on the Si substrate-supported PFO-(PDDA/PSS) 6.5 or PFO-(PDDA/PSS $)_{7}$ film coatings and those after incubation in $1 \mathrm{M}$ $\mathrm{NaCl}$ solution for $20 \mathrm{~min}$. 